\title{
DISCORSO COMMEMORATIVO SU GLSTAVO PIETRO LEJEUNE DIRICHLET
}

PRONUNCIATO

\author{
DA E. E. KLMMER.
}

La importanza scientifica di Lejeune-Dirichlet e di E. E. Kummer, epperò la lusinga di fare cosa grata al pubblico matematico mi determinarono di dare alla stampa la traduzione del discorso commemoralivo pronunciato nella seduta della Reale Accademia delle Scienze di Berlino il 5 Luglio 1860. La mia imperizia, aggiunta alla difficoltà di scostarsi in una traduzione dalla forma straniera, mi obbligano ad invocare l'indulgenza dei lettori, e quella poi specialmente dell'illustre Autore, se lo stile della traduzione è troppo al di sotto dell'originale.

Pavia, Novembre 1860.

\section{Felice Casorati.}

Non sono trascorsi dieci anni da che $\mathrm{i}$ tre uomini; ai quali la nostra patria tedesca va debitrice d'una nuova epoca fiorente per le scienze matematiche, Gauss, Jacobi e Dirichlet, ancor vivevano ed atlivamente adoperavansi a rinnovare e consolidare splendidamente l'antica fama di profonda conoscenza delle verità matematiche si astratte che realizzate in natura, fama che prima di tulti avevano acquistata alla nazione tedesca Keplero e Leibnitz. La nostra Accademia aveva allora la fortuna di possedere come membri attivi due di questi uomini distinti, Jacobi e Dirichlet, i quali, legati da personale amicizia, colla aperta comunicazione dei loro profondi pensieri, si eccilavano e giovavano a vicenda, esercitando la più durevole influenza sullo sviluppo generale delle scienze matematiche. La morte immatura di Jacobi fu la prima perdita irreparabile che colpì la scienza salita in fiore nella nostra patria. L'importanza delle scoperte di questo investigatore di raro ingegno, il posto distinto che terrà per sempre nella storia della matematica vennero dipinti con tanta profondità e verità da Dirichlet, nel discorso commemorativo pronunziato or sono otto anni in questo luogo, ch'egli ebbe per tal mezzo ad erigere alla memoria dell'estinto il più bello e più degno monumento. Allorchè, quattro anni dopo Jacobi, onorò il vecchio Gauss, reputato incontrastabilmente il primo matematico del suo tempo, a questa perdita grave e generale teneva dietro per la nostra Accademia anche la deplorabile conseguenza, che Dirichlel, siccome l'unico degno successore del grand'uomo, era chiamato a Got- 
tinga; cessando cosi di esserne fra i membri presenti. L'Accademia, che non poteva impedire ne riparare tal perdita, colla elezione di Dirichlet a membro effettivo estraneo, riserbavasi il diritto di polerlo considerare ancora come uno de'suoi; quanto poi ai di lui particolari amici di studio, rimase il centro vivente delle loro speculazioni e dei loro lavori, sino a che la morte pose termine alla sua vita ed altività. La nostra Accademia, cui Dirichlet appartenne per ventisette anni, e negli scritti della quale trovavansi depositati gl'immortali capolavori di lui, nel mentre ha il diritto di riguardare come propria la gloria scientifica di questo grande matematico, ha per ciò slesso più di tulli il dovere di conservarne la ricordanza e di tributargli l' estremo onore accademico con pubblica orazione commemorativa. La venerazione che io slesso nutrii costantemente per l'estinto, l'amicizia della quale mi fu largo e che mi conservò per più di venti anni, come pure la stretta relazione fra $i$ miei studj ed $i$ suoi scientifici lavori, mi hanno determinato a prendere la parola al cospetto di questa ragguardevolissima adunanza, per dire della grande importanza scientifica de'suoi capolavori, e delineare nel tempo stesso in pochi tratti l'immagine della sua vita e del suo carattere, il quale era nobile e puro come $i$ suoi scritti. So che non mi sarà dato raggiungere che assai imperfettamente il propostomi intento, non solo perchè la vera importanza delle scoperte intellettuali di grandi uomini non può essere giustamente riconosciuta ed apprezzata che nel successivo corso storico della scienza, in cui le medesime diventano non di rado punti di partenza di teoriche le quali ricevono ampio sviluppo, ma anche per la mia debolezza a motivo di cui io debbo permettermi di far appello alla vostra benigna indulgenza.

Gustavo Pietro Lejeune-Dirichlet nacque il 13 Febbrajo 1805 in Durena. Suo padre, direllore della posta colà, uomo dolce, piacevole ed amabile, e sua madre tuttodì vivente in molto avanzata età, signora spiritosa e finamente educata, diedero al fanciullo, dotato da natura di talenti più che ordinarj, accuratissima educazione. Egli ricevelte la prima istruzione in una scuola elementare, e quando questa non fu più trovala sufficiente per lui, in una scuola privala, dove venne altresì istruito specialmente nella lingua latina, onde potesse in seguito frequentare un ginnasio. La sua grande predilezione per la matematica si palesò assai prestamente, poichè non ancora compiti i dodici anni, impiegava il denaro del suo borsellino nell' acquisto di libri matematici, coi quali si occupava molto assiduamente in ispecie alla sera; e se gli si diceva non poterli comprendere, rispondeva: li leggo tante volte finchè li comprendo. I suoi genitori desideravano che facesse il negoziante, ma avendo egli mostrato decisa ripugnanza per tal carriera, essi cedeltero e lo mandarono nell'anno 1817 al ginnasio di Bonna.

Per amichevole comunicazione del Sig. Prof. Elvenich in Breslavia, che abitava allora in Bonna la stessa casa col giovane Dirichlet, ed al quale era raccomañdata 
caldamente dai solleciti genitori la sorveglianza e direzione del medcsimo, mi trovo in grado di fornire la seguente fedele e viva descrizione del fanciullo, allora in età di circa 13 anni. Distinguevasi ass:i vantaggiosamente nella condolla per bella grazia e buoni costumi, e la ingenuità e schiellezza de'suoi modi facevano sì che lutti quelli i quali trallavano con lui gli erano cordialmente propensi. Le sue cure erano regolate, di preferenza però dirette alla matemalica ed alla storia. Studiava quand'anche non aveva lavori scolastici, allora pure essendo lo svegliato suo ingegno occupato continuamente di argomenti degni di meditazione. I grandi avvenimenti storici, come segnatamente la rivoluzione francese, ed i pubblici affari lo interessavano in alto grado, e giudicava sopra questo ed altre cose con una indipendenza e franchezza di pensare insolito per la sua giovinezza, frutto forse della educazionc ricevuta dai genitori. Qualunque cosa sgarbata ed ignobile eragli contraria, anche $\mathrm{i}$ giuochi però ed altri sollazzi giovanili avevano per lui quasi nessuna altralliva, mentre amava i trattenimenti amichevoli e partecipava volentieri e vivacemente in special modo ai discorsi di politica e di storia. In generale il suo spirito, del quale la dote eminente era l'acutezza, saggirava in una sfera assai più elevata di quello che avvenga solitamente in altri della stessa età.

Al ginnasio di Bonna rimase soltanto due anni, trasferendosi invece a quello dei Gesuiti in Colonia, al quale $i$ suoi genitori diedero la preferenza per motivi a me ignoti. Qui ebbe a maestro nella matematica quel Giorgio Simone Ohm, che divenne poi celebre per la scoperta della legge circa le resistenze alla propagazione delle correnti elettriche, che da lui prese nome; ed in virtù della istruzione che ne ricevette e del proprio assiduo studio di opere matematiche, Dirichlet fece in tale scienza progressi molto rilevanti, procacciandosi estensione straordinaria di cognizioni. Frattanto non trascurò punto le altre discipline e rapidissimamente compì il corso ginnasiale, di modo che nel 1821, all'età di soli sedici anni, otlenne l'altestato per poter entrare nell'Università, e fè ritorno a casa, affine di deliberare co'suoi genitori intorno la scelta della futura carriera. Era ben naturale che questi opponessero alla sua determinazione di studiare matematica il serio avvertimento di assicurare la sua posizione sociale con uno studio più pratico, e come tale gli progettavano la giurisprudenza; però egli dichiarò loro modestamente ma con risolutezza che se lo esigessero li avrebbe assecondati, che per altro non potrebbe abbandonare lo studio prediletto, a cui dedicherebbe almeno le notti. Dietro di che i genitori ragionevoli quanto delicati cedettero al fermo desiderio del figlio.

Lo studio matematico nell'università della Prussia e della rimanente Germania era allora in miserrimo stato. Le lezioni elevandosi di poco al disopra della sfera della matematica elementare erano affatto insufficienti ad appagare lo stimolo di cognizioni più profonde, che animava il giovane Dirichlet, non eravi in Germania, fuori dell' 
unico gran nome di Gauss, niun'altro che avesse potulo esercitare su di luì una speciale attrazione. In Francia allincontro e segnatamente in Parigi, la matematica era ancora nella sua piena floridezza, ed un circolo di uomini, il gran nome de'quali risplenderà eternamente nella storia delle matematiche, si adoperava coll'investigazione e coll'insegnamento a svilupparle e diffonderle efficacemente. Quivi peranco viveva il grande Laplace, al quale la Meccanica Celeste assicurava incontrastabilmente il primo posto, lavorando tultora al compimento di quest'opera e ad un supplemento della sua teorica della probabilità. Legendre, sino alla avanzata età indefessamente operoso, stava perfezionando la sua teorica delle funzioni ellittiche, colla scoperta di una nuova trasformazione delle medesime, e preparando la terza edizione dell'opera sulla teorica dei numeri. Fourier, che da poco tempo aveva terminata la teorica matematica del calore, riuniva intorno a se in scientifiche e gioviali conversazioni un' eletta schiera dei giovani matemalici più ingegnosi. Poisson arricchiva la Mcceanica e la Fisica matemalica di una serie di memorie pregiatissime. Cauchy poneva allora i fondamenti per migliorare e riformare radicalmente tutta quanta l'analisi, con metodi più rigorosi e colla introduzione delle variabili immaginarie. Questi uomini non che un ragguardevole numero di altre scientifiche notabilità, delle quali alcune vivono tuttora, cooperavano a rendere Parigi la più splendida sede delle scienze matematiche.

Giustamente apprezzando tali circostanze, Dirichlet riconobbe ch'era questo il sito, d'onde poteva aspeltarsi pe'suoi studj matematici il maggiore profitto, ed acconsentendovi di buon grado i suoi genitori, i quali per mezzo di alcune famiglie amiche erano da molto tempo in relazione con Parigi, egli recossi nel Maggio del 1822 a codesta scuola superiore delle matematiche discipline, colla lieta coscienza di potersi ormai dedicare completamente allo studio prediletto. Quivi frequentò le lezioni al Collegio di Francia ed alla Facoltà delle Scienze, dove ebbe a maestri Lacroix, Biot, Hachette e Francoeur. Un tentativo da lui falto di poter assistere anche alle lezioni alla Scuola Politecnica come ospitante andò fallito, perchè l'incaricato d'affari prussiano a Parigi non volle assumersi, senza speciale autorizzazione del Ministro di Altenstein, di chiederne il permesso al Ministro francese.

Oltre di assistere alle lezioni e di meditare sugli argomenti in esse esposti, il Dirichlet dedicava il tempo anche all'attento studio delle migliori opere matematiche, e fra queste specialmente al lavoro di Gauss intorno l'Aritmetica superiore: Disquisitiones Arithmeticae. Siffatto lavoro esercitò su tulta la educazione e sull'indirizzo matematico di lui un' influenza assai più rilevante che non gli altri suoi studj di $\mathbf{P a -}$ rigi; non una nè parecchie volte egli ebbe a studiarlo, ma per tutta la vita non cessò mai con ripetuta lettura di richiamarsi alla mente la copia dei profondi pensieri in esso contenuti, per cui il medesimo non trovavasi mai nello Scaffale, ma aveva un posto fisso sul tavolino di lavoro. Quale sforzo gli abbia dovuto costare l'addentrarsi 
in quest'opera straordinaria, si può desumere da ciò che più di venti anni dopo la sua comparsa, nessuno peranco dei matemalici allora viventi l'aveva studiata e compresa interamente, e che Legendre stesso, il quale aveva dedicato gran parte della sua vita all'Aritmetica superiore, dovelle confessare nella seconda edizione della sua teorica dei numeri, che avrebbe volentieri arricchito il suo lavoro dei risultati di Gauss, ma i metodi di questo autore essere si originali da non poterli riprodurre senza enormemente dilungarsi od assumere la parte di semplice traduttore. Dirichlet fu il primo non solo a comprendere perfeltamente tale lavoro, ma anche ad aprirne l'adito ad altri, rendendo facili e chiari i rigidi metodi, dietro i quali stavano celati i profondi pensieri, e scambiandoli in molti punti principali con altri più semplici e più conformi alla genesi, senza rinunciare menomamente al perfeṭlo rigore delle dimostrazioni; egli fu eziandio il primo che oltrepassandone i confini abbia manifestato un ricco tesoro di segreti ancor più profondi nella teorica dei numeri.

La vita esteriore di Dirichlet nel primo anno di soggiorno in Parigi era sommamente semplice e ritirata. I suoi studj, interrotti una sola volta in causa di un' attacco di vajuolo, lo assorbivano completamente, limitandosi egli a frequentare alcune case, alle quali era raccomandato, ed a trovarsi con alcuni giovani tedeschi, ivi dimoranti per istudiare. Ma nell' estate del 1823 sopravvenne un cambiamento della più grande importanza per la intiera sua educazione. Il generale Foy, uomo pien di spirito e di molteplice cultura, non meno distinto per l'alto posto che teneva come capo dell'opposizione nella camera dei deputati e come uno de'più celebri oratori della medesima, quanto per la brillante sua carriera militare, e la cui casa era una delle più ragguardevoli ed ambite in Parigi, cercava allora un giovane a maestro de'suoi figli, il quale li avesse ad istruire principalmente nella lingua e letleratura tedesca; per tale ufficio fu raccomandato al generale il nostro Dirichlet, da un'amico di casa Dirichlet, il Sig. Larchet de Chamont. Presentatosi per la prima volta, le sue maniere aperte e morleste fecero sul Generale impressione sì favorevole, che gli affidò tosto l'ufficio di maestro dei figli, con stipendio conveniente ed obblighi tanto lievi, da rimanergli libertà di tempo bastante per continuare gli studj intrapresi. Dirichlet assunse con giubilo siffatto incarico, non solo perchè veniva messo in posizione di non cagionare ulteriori spese a'suoi genitori, ma principalmente anche perchè dalla dimora in casa d'uomo sì perfeltamente educato e distinto ripromellevasi molto per la propria educazione esteriore, nella quale a suo proprio giudizio era peranco assai indietro. In questa nuova posizione egli sentivasi straordinariamente contento e felice, dappoichè i signori conjugi Foy dimostravangli in ogni cosa la più grande amorevolezza e cortesia, considerandolo qual membro della loro famiglia. L'istruzione dei figli, dei quali una fanciulla di undici anni era la maggiore, costavagli poca fatica; la moglie poi del generale che, addistrata nella sua fanciullezza nella lingua tedesca, Tom. III. No 4. 1860 . 
avendola in seguito completamente dimenticata, tornó ora sotto la sua direzione a coltivarla diligentemente e col miglior successo, lo ricompensava della fatica in modo tanto aggradevole quanto utile, correggendogli, mediante esercizj di lettura in lingua francese, l'accento straniero della pronuncia. La più grande influenza su di lui l'esercitava però il Generale, coll' esempio vivente di uomo operoso, nobile e finamente educato, e questa influenza non estendevasi soltanto alla esteriore educazione di Dirichlet, alle sue abitudini ed inclinazioni, sibbene anche al suo modo di pensare e di agire ed a tutte le contingenze della vita. Di grande importanza per tutta la sua vita fu pure che la casa del Generale, punto di riunione delle prime notabilità scientifiche ed artistiche della capitale francese, e nella quale venivano trattate dai più ragguardevoli membri della camera le grandi questioni politiche, la cui prossima e preliminare soluzione seguiva nell'anno 1830 , gli diede per la prima volta occasione di vedere la vita in grande scala e di interessarvisi.

Da tulte queste nuove impressioni che riceveva, dalle occupazioni e distrazioni che andarono congiunte al suo posto, Dirichlet non lasciavasi punto deviare da'suoi studj matematici, anzi precisamente in questo tempo preparava con fervido zelo iI primo scritto dato alla luce : Mémoire sur l'impossibilité de quelques équations indéterminées du cinquième degré. L'argomento di questa memoria ha il più strelto rapporto col teorema di Fermat, che la somme delle eguali potenze di due numeri non può mai essere eguale ad una sola potenza dello stesso ordine delle precedenti, ove queste potenze siano superiori alla seconda. Siffatto teorema, cui Fermat asserisce di poter dimostrare, che però sino oltre 150 anni dopo Fermat, nel tempo in cui Dirichlet se ne occupava, non ostante i più grandi sforzi di Eulero e Legendre, non aveva potuto dimostrarsi più in là della terza e quarta potenza, siffatto teorema, come particolarità tolta dalla propria concatenazione scientifica, non ha veramente diritto ad alcun valore speciale; tuttavia ha conseguito una importanza straordinaria per ciò che essendo un punto di mira apparentemente vicino ma in effetto ancora lontano innalzato nel campo delle forme dei gradi superiori allora peranco sconosciuto, esercitò la più decisiva influenza sullindirizzo preso dalla teorica dei numeri nel proprio sviluppo storico. Dirichlet, seguendo nel suo lavoro la via segnata dal teorema di Fermat, considerava la somma di due quinte potenze, circa le quali nulla ancora si era trovato, e si propone in pari tempo la questione un po'più generale, di ricercare cioè in quali casi tale somma non possa essere eguale ad un multiplo dato di una quinta potenza. Egli rendesi piana e sicura la via della ricerca, mediante alcuni teoremi intorno la soluzione la più generale della questione : rendere una forma quadratiça eguale ad una potenza d'un numero, e riesce a dimostrare la impossibilità di una classe intiera di equazioni del quinto grado. La equazione di Fermat per le quinte potenze, delle quali una necessariamente dovrebb'essere divisibile per cin- 
que, è soltanto nel caso in cui questa sia anche pari che trovasi compresa nella classe suddelta; tultavia l'altro caso, in cui la medesima sia dispari, fu similmente dimostrato impossibile poco tempo dopo da Legendre, che si avanzò un po' più ancora nel cammino aperto da Dirichlet. L'onore portanto di avere spinta d'un intiero gradino più in là la dimostrazione di questo teorema storicamente rimarehevole va diviso fra Dirichlet, e Legendre.

Non soltanto i nuovi risultati ottenuti in una delle parti più difficili della teorica dei numeri, ma eziandio la forza e solligliezza delle dimostrazioni e la singolare chiarezza della esposizione assicuravano a questo primo lavoro di Dirichlet un brillante successo. l'Accademia di Parigi, a cui lo presentò gliene accordava la lettura nella seduta dell'undici giugno 1825, e tantosto nella seduta susseguente, del diciotto di detto mese, i Sigg. Lacroix e Legendre ne presentavano quali commissari un rapporto si favorerole, che l'Accademia decideva di farlo inserire nelle raccolta delle memorie dei dotli stranieri. La fama di Dirichlet come matematico distinto veniva perciò stabilita, c d'allora in poi come giovane, dal quale attendevasi uno splendido avvenire, fu nei più elevati circoli scientifici di Parigi non solo ammesso ma anche cercato. Egli entrò per tal guisa in più stretta relazione con parecchi dei più ragguardevoli membri dell'Accademia di Parigi, fra i quali debbonsi rimarcare specialmente due, cioè Fourier ed Alessandro di Humboldt, che esercitarono molta influenza, il primo sull' indirizzo delle sue investigazioni scientifiche, il secondo sullo sviluppo successivo della sua vita esteriore.

Fourier, il quale dalla propria giovinezza, in cui erasi altivamente adoperato alla fondazione della Scuola Normale e della Scuola Politecnica, conservava ancora intatto l'entusiasmo per una animata corrispondenza scientifica, e pel quale era un'intimo bisogno il comunicare anche a viva voce quanto di bello e di grande aveva investigato, trovò in Dirichlet un giovane, cui poter aprire completamente il suo cuore matematico, e dal quale era non soltanto ammirato, ma anche perfettamente compreso. Lo introdusse quindi nel circolo dei distinti giovani matematici, che soleva riunire intorno a se, coi quali s'intratteneva nei modi vivaci ed attraenti propri di lui sulla sua teorica del calore, e sopra i suoi nuovi metodi analitici a tale scopo trovati, come pure sopra ogni specie di argomenti e questioni più generali nella scienza. In questo circolo, al quale apparteneva fra gli altri anche Sturm, divenuto poco dopo celebre - pel teorema sopra le radici delle equazioni algebriche, Dirichlet ricevette molteplici eccitamenti, ma segnatamente da Fourier fu promosso il suo interesse per la fisica matematica, nella quale lavorò in seguito con rilevante successo; così pure ne' suoi successivi lavori prendono un posto non indifferente la serie e gl'integrali di Fourier, che debbono ai metodi rigorosi di Dirichlet il loro vero fondamento scientifico.

Alessandro di Humbold allora dimorante in Parigi, aveva gia dapprima dal Ge- 
nerale Foy udito lodare Dirichlet come matematico distinto, ma senza attribuire molto peso all'elogio, che non sortiva dalle labbra di uomo versato nella materia; e però non lo conobbe più davvicino se non quando Dirichlet presentossi a fargli visita, dietro la favorevole accoglienza fatta al suo scritto dall'Accademia. Humboldt lo accolse colla più squisita amorevolezza e cortesia, attestandogli colla stima pel suo talento, e la sua abilità scientifica anche il più vivo interessamento ed affetto, che d'allora in poi gli mantenne incessantemente, e gli provò coi fatti. Sino da quesia prima visita Dirichlet diede a conoscere nel discorso la intenzione di fare in seguito ritorno in patria, ed Humboldt, accogliendo con gioja tale pensiero, lo rafforzò nel proposito coll'assicurazione, che, stante l'estrema scarsezza di valenti matematici tedeschi, non avrebbe potuto mancargli una eccellente posizione, tostochè l'avesse desiderata. Nelle condizioni di quel tempo, mentre appunto eransi dovute abbandonare le pratiche proseguite parecchi anni onde far venire Gauss a Berlino, per poche centinaja di talleri, siffatta assicurazione non era facilmente adempibile, e poco dopo ci volle la instancabile altività e tutta quanta la influenza di Humboldt per rializzarla anche solo approssimativamente.

Per la morte avvenuta nel Novembre del 1825 del suo proteltore sommamente venerato, il Generale Foy, e per la influenza di Alessandro di Humboldt, che abbandonò dopo poco tempo Parigi onde stabilirsi in Berlino, la risoluzione di Dirichlet di far ritorno in patria venne a maturanza. Indirizzó al Ministro di Altensteir una istanza per ottenere un collocamento conveniente, istanza che Humboldt prese ad appoggiare, e rendere efficace mercè la propria influenza; e ritornò nell'autunno del 1826 a Durena presso i suoi genitori, onde aspettarne l'esito.

Mentre quivi attendeva ad una nuova memoria, di cui avrò quanto prima a parlare, Humboldt ne promoveva col più gran zelo il collocamento. Affine di ottenere per Dirichlet un posto di professore straordinario presso qualche università prussiana, collo stipendio di sei ad oltocento talleri, si rivolse personalmente al Ministro di Altenstein, interessò a tale intento i più ragguardevoli membri della nostra Accademia, rinforzando così la propria raceomandazione colla loro, e diede a Dirichlet relazioni frequenti e buoni consigli su ciò che per parte sua dovesse fare, con tulte queste premure peró, che Gauss istesso appoggiò mediante uno scrilto direlto al nostro collega Sig. Encke, e da esso consegnato al regio ministero, altro non si potè tultavia ottenere se non che gli fossero assicurati annualmente 400 talleri come rimunerazione fissa, onde potesse abilitarsi a Breslavia nella qualità di docente privato. Tale rimunerazione assicurandogli tosto una discreta sussistenza e potendo far conto che Humboldt avrebbe continuato ad adoperarsi per procacciargli una posizione più conveniente, acceltò senza difficoltà. Frattanto honoris causa era anche stato elelto doltore di filosofia della facoltà filosofica dell'universilà di Bonna, la quale cosa gli rese assai più facile l'abilitazione ad una università. 


\section{PURA ED APPLICATA.}

Nel recarsi a Breslavia scelse la via per Gottinga, affine di conoscere Gauss personalmente, al quale si presentò il 18 di Marzo del 1827. Notizie più dettagliate di tale incontro non mi fu possibile avere; una lettera d'allora diretta alla madre di lui altro non dice che Gauss lo accolse molto amichevolmente, e l'impressione personale di questo grand'uomo fu assai più favorevole di quello che si aspetlasse.

Ora in Breslavia, secondo gli statuti di quella facoltà filosofica, per ottenere la venia docendi, doveva fare una lezione di prova, tenere un discorso innanzi alla facoltà, scrivere una dissertazione, e difenderla pubblicamente in lingua latina. A tali esigenze, in quanto riferivansi alla sua scienza era più che idoneo, sapeva altresi scrivere benissimo chiaramente e corretlamente latino sopra un'argomento scientifico, ma non aveva mai impiegato il suo tempo, destinato a più elevati scopi scientifici, nel procacciarsi la disinvoltura della favella latina; la vuota formalita della disputa latina lo disturbava e gli spiaceva quindi in alto grado. Con sua grande soddisfazione, ma con grande cordoglio di alcuni signori di quella facoltà, dopo ch'ebbe tenuto la lezione di prova sulla irrazionalità del numero $\pi$, venne dal regio ministero dispensato dalla pubblica disputa, ed affinchè potesse subito cominciare le lezioni, senza delle quali la scienza matemalica sarebbe quivi rimasta quasi senza alcuna rappresentanza, oltenne in pari tempo il permesso di non presentare che posteriormente il suo scritto latino di abilitazione.

Il successo dell'attività sua nell' istruire nei tre semestri duranti i quali insegnò in Breslavia non fu rilevante. Gli studenti di quel sito, non spingendosi volentieri oltre l'angusta sfera di idee matematiche loro insino a quel tempo somministrate, non potevano abituarsi tanto facilmente alla sua maniera d'insegnare, per essi straniera, di più il suo modo di presentarsi modesto e persino un po'peritoso non era falto per imporre loro. In generale Dirichlet in Breslavia era bensì veduto di buon grado e cercato in tutte le socielà, come giovane finamente educato ed amabile, ma come matemalico era poco apprezzato in confronto del suo predecessore, il quale aveva scritto un trattato di Geometria analitica. Non parlando mai di se, e de' suoi meriti scientifici, ne avendo alcun appoggio letterario, che ciò si assumesse a suo favore, così non raggiunse ivi quella celebrità locale o provinciale, che in circoli ristrelli, e più efficace che l'essere universalmente riconosciuto da parte dei primi uomini della scienza.

Durante la sua dimora in Breslavia, Dirichlet scrisse due memorie, che ebberc entrambe origine dalla memoria di Gauss sui residui biquadratici. Le gelehrten Auzeigen di Gotlinga dell'Aprile 1825 avevano dato un succinto annuncio di una serie di memorie sopra i residui biquadratici e le loro leggi di reciprocità, che Gauss pensava di pubblicare, delle quali la prima era altresi già presentata alla Società delle Scienze di Goltinga, ma non veniva alla luce che tre anni dopo. Tale annuncio, che esponeva alcuni dei risultati di Crauss, le dimostrazioni dei quali dovevano basarsi s0- 
pra un principio affatto nuovo della teorica dei numeri, eccitó in alto grado la sete di sapere simultaneamente di Jacobi e Dirichlet; entrambi cercarono per vie affatto diverse di penetrare nel segreto di Gauss, e ad entrambi pure riuscì di trovare in questo campo dei residni di potenze superiori una moltitudine di progressioni nuove, sebbene il nuovo principio, consistente nella introduzione dei numeri interi complessi, rimanesse loro ancora nascosta. Dirichlet trovò pei teoremi di Gauss già pubblicati, contenenti la completa soluzione della questione : " assegnare tutti $i$ numeri primi pei quali il numero due è residuo o non residuo biquadratico " trovò dimostrazioni di una semplicità meravigliosa, coi metodi noti della teorica dei numeri, e nello stesso modo risolvette anche la questione più generale per qualsiasi numero primo dato, così che onde giungere alla completa legge di reciprocità per i residui biquadratici, rimaneva aneora un solo passo a fare, il quale però non divenne possibile che col nuovo principio di Gauss. Nell'altro scritto allora pubblicato, che Dirichlet stese in lontano e presentò alla Facoltà Filosofica come suo scritto di abilitazione, fornisce un'esempio allora affatto nuovo di forme di gradi comunque elevati, i divisori delle quali hanno determinate forme lineari. I risultati di questo lavoro possono presentemente risguardarsi come casi parlicolari dei teoremi generali sopra i divisori delle norme dei numeri complessi formati da radici délluniti.

Per dare un saggio del merito, che si attribuiva allora segnatamente al primo di questi due lavori, addurrò i giudizj sul medesimo di Bessel e Fourier. Bessel scrive in una lettera ad Humboldt : Chi avrebbe pensato che al genio sarebbe riuscito di ridurre a considerazioni tanto semplici cose di apparenza si difficile; sulla memoria potevasi trovare il nome di Lagrange che nessuno ne avrebbe rimarcata la falsità. Fourier invece pose i risultati di Dirichlet persino al di sopra delle grandi scoperte di Jacobi e di Abel nella teorica delle funzioni ellitliche, delle quali per verità egli non aveva avuto conoscienza sc non pegli elogi di Legendre, che riteneva eccessivi. In una lettera diretta a Dirichlet, come pure in manifestazioni a voce fatte all'amico su citato della famiglia Dirichlet, il Sig. Larchet de Chamont, dalle quali è tolta la sentenza riferita, esprime anche il vivo desiderio, che Dirichlet avesse a ritornare a Parigi, siccome destinato a presto occupare uno dei primi posti in quell'Accademia.

Frattanto Alessandro di Humboldt aveva oltenuta la nomina di Dirichlet a professore straordinario all'universilà di Breslavia, e poscia si adoperara di guadagnarlo a questa Università ed all'Accademia, ed anzitutto insomma di farlo venire a Berlino. Un posto d'insegnamento matemalico, che diveniva vacante alla scuola militare generale, avendone fornita l'opporlunità, Humboldt ne approfittò per raccomandare assai caldamente Dirichlet al generale di Radowitz ed al ministro della guerra. Costoro tultavia non potevano decidersi subito ad affidargli definitivamente la carica, verosimilmente perchè allora non contando che 23 anni poteva sembrare troppo gio- 
rane; perciò si fece in modo presso il ministro di Altenstein che Dirichlet ottenesse tosto il permesso di un'anno, affine di assumere provisoriamente l'insegnamento alla scuola militare.

Nell'autunno del 1828 egli veniva a Berlino onde entrare nella nuova carica. Le lezioni di maltematica che doveva dare ad ufficiali circa della sua elà gli facevano molto piacere, la relazione con militari educati, ai quali era avvezzo sino dalla sua dimora nella casa del generale $\mathbf{F o y}$, eragli assai gradita, e siccome in quel tempo aveva fatto fra gli altri anche studj fondamentali nella moderna storia militare, cosi oltre la matematica era vincolato a'suoi uditori eziandio da questo comune interesse. E non fu che più tardi, dopo essersi formato a questa università un gran circolo di uditori che lo seguivano col maggiore interessamento scientifico nelle più alte sfere della matematica, nelle quali egli preferiva sommamente di aggirarsi, che svegliossi in Jui it desiderio di essere esonerato dall'insegnamento alla scuola militare, il quale desiderio fu poi uno dei principali motivi della sua traslocazione a Gottinga.

Non appena giunto in Berlino Dirichlet fece anche i passi necessarj da poter dare lezioni presso questa università. Come professore di un' altra università non vi era autorizzato; non gli rimase quindi se non che di farsi abilitare nuovamente come docente privato, ed in tali sensi presentò istanza alla facoltà filosofica. La quale gli rilasciò il decreto di abilitazione in vista della sua abilità scientifica altrove provata, e così cominciò a dare lezioni sotto il titolo di docente privato. Il suo collocamento definitivo presso questa università, come professore straordinario, non segui che nel 1831, e dopo alcuni mesi fu eletto dalla nostra Accademia a membro ordinario. Nello stesso anno si unì in matrimonio con Rebecca Mendelssohn-Bartholdy nipote di Mosè Mendelssohn, ed in ciò pure ebbe parte notabile, ma involontaria Alessandro di Humboldt, siccome quegli che introdusse per la prima volta Dirichlet nclla famiglia dei genitori della moglie, famiglia distinta e rinomata per spirito e talento artistico.

I casi successivi della vita scompajono ora per lungo tempo di fronte allimportanza dei lavori scientifici da lui eseguiti, durante i 27 anni che visse in questo luogo. Nella descrizione che ora m'incombe di farne, tenterò di esporre a grandi tratti ed in. generale i progressi scientifici in essi contenuti, considerandoli non isolatamente a seconda del tempo di loro origine, ma aggruppati a norma dell'argomento e delle idee che loro servono di base. 